\title{
Distinguishing P-N Junction and Schottky Barrier in a Working Silicon Nanowire Diode
}

\author{
Kai He ${ }^{1}$, Jeong-Hyun $\mathrm{Cho}^{2}$, Yeonwoong Jung ${ }^{3}$, Mark Reed ${ }^{3}$, Tom Picraux ${ }^{2}$ and John Cumings ${ }^{1}$ \\ 1. Department of Materials Science and Engineering, University of Maryland, College Park, MD 20742 \\ 2. Center for Integrated Nanotechnologies, Los Alamos National Laboratory, Los Alamos, NM 87545 \\ 3. Department of Electrical Engineering and Applied Physics, Yale University, New Haven, CT 06520
}

Silicon nanowires (SiNWs) have attracted tremendous attention over the past decade because of their unique electronic properties and promising applications as building blocks for nanoscaled photonic and electronic devices, as well as for emerging sustainable energy technologies, i.e. photovoltaic solar cells and lithium ion batteries [1,2]. Regardless of which specific application, SiNWs must be interfaced with other materials and operated at different electrical potentials, where the contact properties are critical to the overall functionality of the devices [3]. It is thus of great importance to understand the underlying science of contact formation and use it to precisely tailor proper electrical contacts on the nanoscale.

In this work, we report an in-situ examination of individual p-n junction SiNWs using off-axis electron holography (EH) during transmission electron microscopy (TEM) [4]. The free-standing SiNWs were synthesized onto ${ }^{+}-S i$ substrates by chemical vapor deposition (CVD) with an axial dopant profile from $\mathrm{n}$ - to $\mathrm{p}$-type, and then placed inside TEM in a cantilevered geometry in contact to a movable Pt probe for in-situ biasing measurements during simultaneous TEM or EH observations, as shown in Figure 1 (a). The phase shift from EH (Figure 1 (c)) indicates a potential shift between the p- and n-segments to be $1.03 \pm 0.17 \mathrm{~V}$ due to the built-in voltage, as well as a depletion width of $287 \mathrm{~nm}$. However, the I-V characteristics of this SiNW display inverted rectification behavior for the expected p-n junction, indicating the formation of a Schottky barrier between the NW tip and the Pt contact, as indicated in Figure 2. Corresponding EH phase profiles from the NW tip to the NW base under applied bias of $\pm 3 \mathrm{~V}$ are plotted in Figure 2. The large phase shifts at the NW tip indicate a strong concentration of electric field at this Schottky contact, preventing any change of the Si energy bands in the p-n junction region by the applied bias.

In summary, we have justified a successful approach to study electrostatic potential distribution in and around a working device on the nanoscale by combination of in-situ TEM and EH techniques. The p-n junction properties are quantitatively measured, and consistent with the theoretical calculations, but its transport behavior is dominated by the Schottky barrier at the probe contact. From device functionalization point of view, this type of mechanical point-contacting does not provide stable electrical connection with good ohmic-contact performance, due to the strong influence of a metalsemiconductor Schottky barrier. Nevertheless, the results will provide valuable information for device design and fabrication via a bottom-up strategy [5].

\section{References:}

[1] Y Cui and C M Lieber, Science (2001) 291, 851.

[2] C K Chan et al, Nature Nanotechnology (2008) 3, 31.

[3] F Léonard and A A Talin, Nature Nanotechnology (2011) 6, 773.

[4] K He et al, Nanotechnology, (2013) in press.

[5] The authors acknowledge funding from US DOE-EFRC under Award Number DESC0001160. 


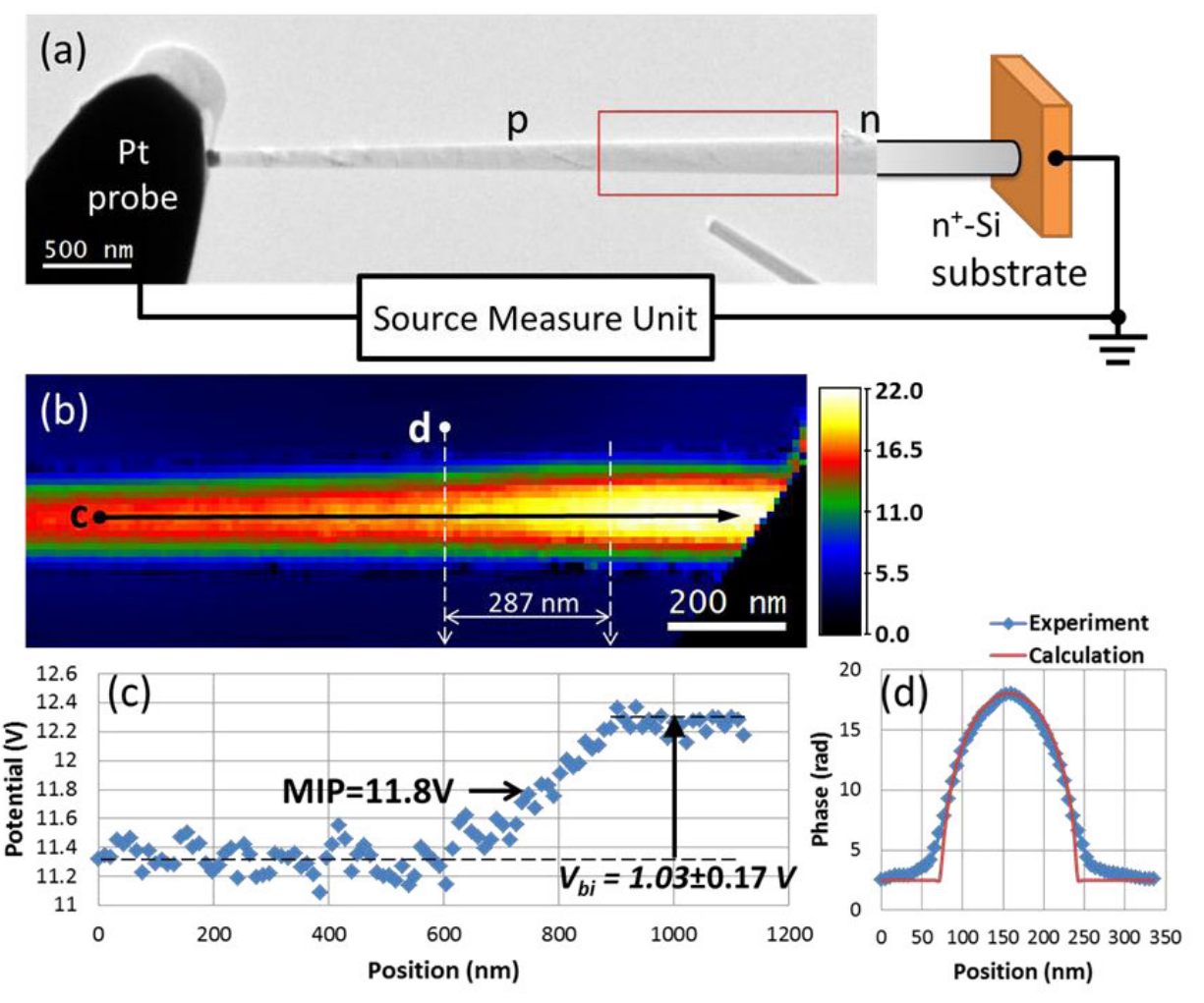

Figure 1. (a) Schematics of experimental setup, with a TEM image showing a SiNW in point-contact to the Pt probe. (b) Electron holographic phase image of the SiNW p-n junction in the boxed region. (c) Potential profile corresponding to the arrow c shows a built-in potential shift of $1.03 \pm 0.17 \mathrm{~V}$ across the p-n junction. (d) Phase profile along the arrow d showing good agreement with a model calculation.

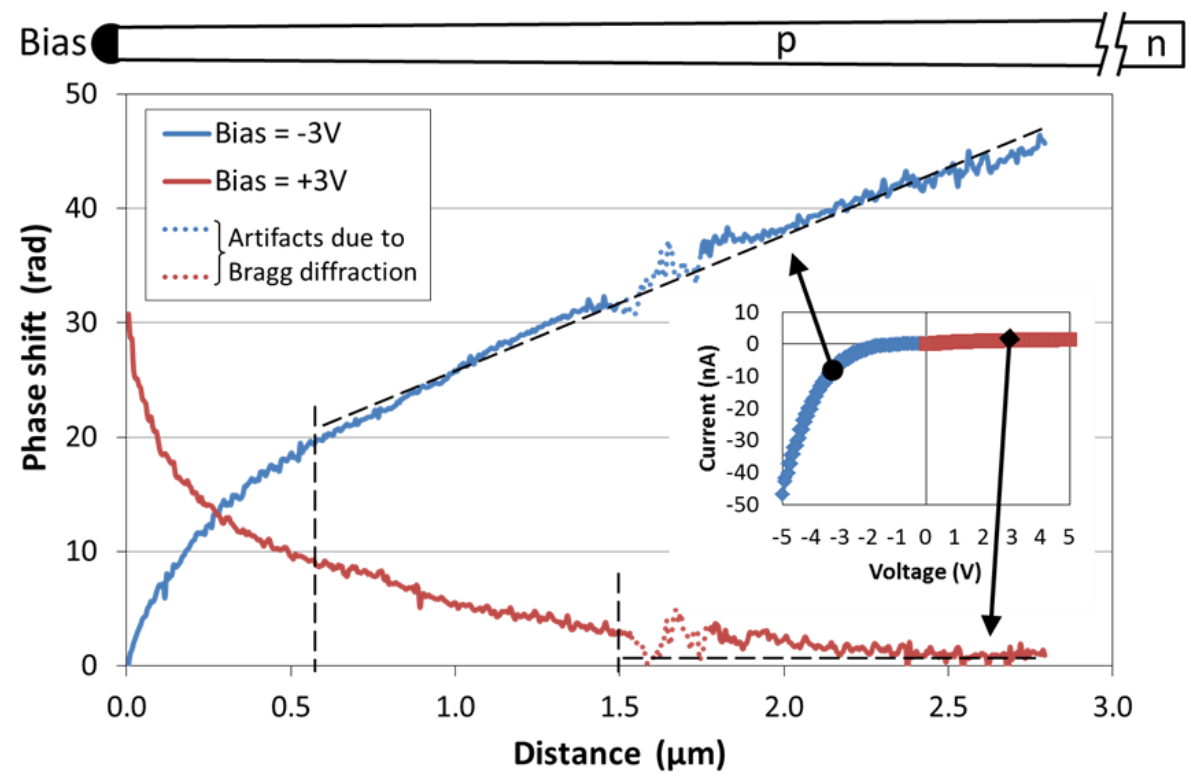

Figure 2. Phase profiles along the SiNW from Au tip toward NW base under applied bias of $\pm 3 \mathrm{~V}$ to the NW tip. Dotted lines indicate artifact data points due to strong Bragg diffraction. Dashed lines are guides to the eye, indicating trends of the phase profile and the approximate depletion widths of the Schottky barrier. Inset I-V curve showing the rectification behavior due to the Schottky barrier. 